\title{
Application-Aware Video Coding Architecture Using Camera and Object Motion-Models
}

\author{
R.M.T.P. Rajakaruna, W.A.C. Fernando, Member, IEEE and J. Calic, Member, IEEE,
}

\begin{abstract}
The proliferation of video consumption, especially over mobile devices, has created a demand for efficient interactive video applications and high-level video analysis. This is particularly significant in real-time applications and resourcelimited scenarios. Pixel-domain video processing is often inefficient for many of these applications due to its complexity, whereas compressed domain processing offer fast but unreliable results. In order to achieve fast and effective video processing, this paper proposes a novel video encoding architecture that facilitate efficient compressed domain processing, while maintaining compliance with the mainstream coding standards. This is achieved by optimizing the accuracy of motion information embedded in the compressed video, in addition to compression efficiency. In a motion detection application, we demonstrate that the motion estimated by the proposed encoder can be directly used to extract object information, as opposed to conventionally coded video. The incurred rate distortion overheads can be weighed against the reduced processing required for video analysis targeting a wide spectrum of computer vision applications.
\end{abstract}

Index Terms-Feature Extraction, Motion Detection, Vision Applications, Video Analysis

\section{INTRODUCTION}

Video processing plays a vital role in a wide range of applications such as surveillance, automation and control systems, content-based retrieval, and multimedia classification. Many of these applications require efficient handling of large amounts of data, often in real-time [1] - [2]. As a result, processing in pixel domain, which is computationally intensive, is not effective in many scenarios. The inherent complexity could be minimized by using auxiliary information about video content, i.e. metadata. Delivering metadata separately from the video stream requires an application dependent architecture that would incur additional system complexity. Instead, information embedded in the encoded bit-stream, such as motion vectors (MVs), transform coefficients and block prediction modes - information often referred to as compressed domain features - can be used as auxiliary information. For example, Babu et al. [3], Mezariz et al. [4] and Porikli et al. [5] present different methods of using MVs and transform coefficients in video segmentation. Variations of block prediction modes are used to filter motion information by Bruyne et al. [6], to identify regions-of-interest in image sequences. These methods

R.M.T.P. Rajakaruna, W.A.C. Fernando and J. Calic are with the with the I-Lab Multimedia Communications Research, at the Centre for Vision, Speech and Signal Processing, University of Surrey, UK. (+441483683749; e-mail: r.rajakaruna,w.fernando,j.calic@ @urrey.ac.uk)

This work has been supported by the MUSCADE Integrating Project, funded under the European Commission ICT 7th Framework Program. are more economical in processing complexity, at the expense of output precision, in contrast to pixel domain methods.

The performance of compressed domain processing depends on the accuracy of compressed domain features in representing video content. However, compression-efficiency driven nature of encoder parameter selection often leads to sparse and noisy compressed domain features. Therefore, while compressed domain video processing reduces complexity, this is achieved at the expense of output precision and reliability. In order to achieve fast video processing and yet improve the reliability of the analysis, this paper proposes a novel application driven video encoding architecture. By steering encoder parameter selection towards accurate content description, this proposal enhances reliability of compressed domain features without altering the coded data structure. Therefore, while any compressed domain application would benefit, decoder and application architectures are not affected.

Application-aware video coding methods have been proposed in literature in [7], [8] and [9]. Enforced H.264 blocksize selection is used by kapatos et al. [7], to demarcate scene changes. This requires a modified decoder and change-aware applications. kas et al. [8] proposes background MV correction in the encoder to improve motion based video indexing. These methods each support only a given application. In [9], we proposed a content driven motion selection mechanism, independent of compression constraints, to enhance performance of applications based on object motion. However, it is limited to identifying singular motion. In Addition, compression requirements should be considered in parallel with application requirements to provide a flexible solution.

In this work, we integrate rate-distortion (RD) constraints of conventional encoding with the requirements of motion-based vision applications. This is achieved by extending the encoder parameter selection process with a novel objective function that represents accuracy of motion vectors. Each frame is pre-processed to learn object and camera motion to generate reference information to steer the objective function towards accurate content description. We use object boundaries and spatial correlation of motion to accommodate multiple moving objects and camera motion. By taking moving object detection and segmentation as an example, we demonstrate end-to-end implementation of the proposed architecture.

The rest of the paper is organized as follows. Problem background is discussed in Section II and the proposed applications-aware video coding architecture is presented in Section III. Section IV outlines experimental results, while applications of the proposed video coding architecture and the conclusion are given in sections V and VI respectively. 


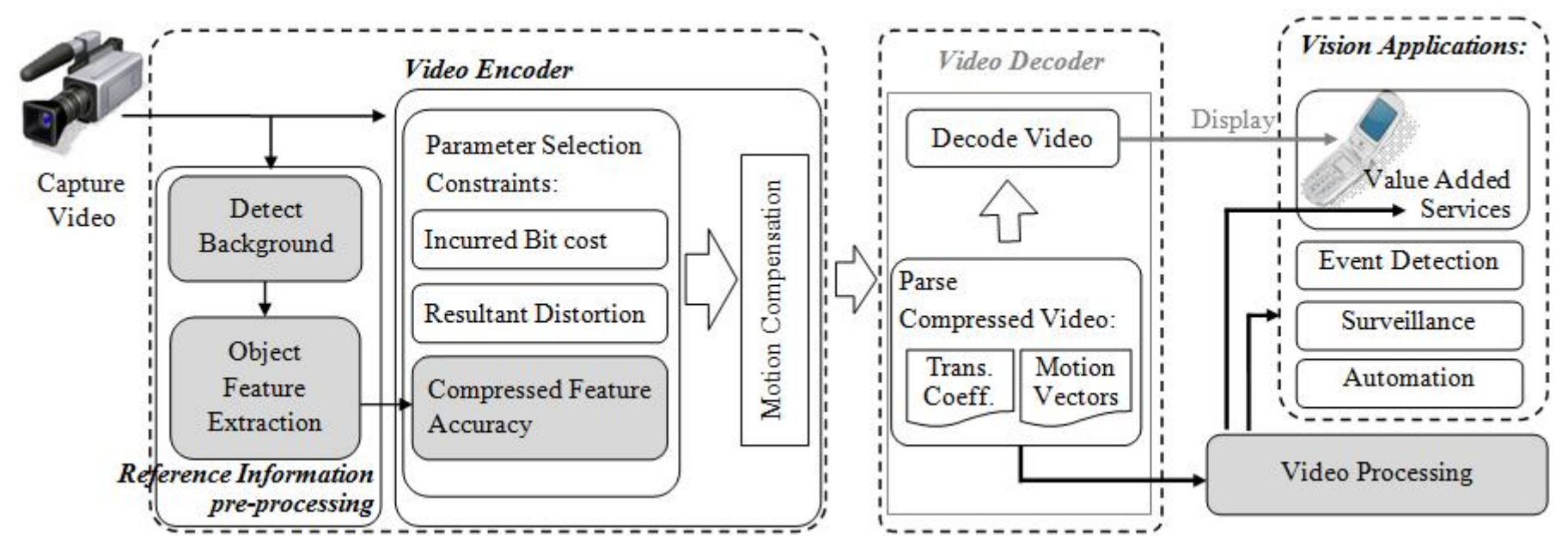

Fig. 1. Overview of the proposed application-aware video coding architecture

\section{SCOPE AND PRoblem BACKGROUND}

In the compressed domain feature set, MVs are evaluated independently, whereas residual data depend on selected MVs. Therefore, we limit the focus of this work to motion information present in the compressed domain.

Existing video coding standards use motion modelled by 2D translational MVs in inter-frame prediction. Each frame is divided into blocks, and each block is assigned a separate MV to generate a predicted frame. These MVs collectively represent spatial and temporal distribution of frame level activity as well as local motion. Therefore, we selected Object detection as the application scenario since it utilizes both local and frame level activity. Additionally, segmentation and extraction of object information is the base of many video applications, from surveillance to interaction.

Conventionally, motion selection problem in the encoder, is formulated as an optimization, where resultant distortion is minimized subjected to maximum number of bits allowed in encoding a given coding unit. This ensures compression efficiency, which is critical in video communication. However, other aspects of video utilization, such as video analysis and its applications, would benefit from enhanced quality of information embedded in the compressed video stream.

\section{ApplicAtion-Aware Video Coding ARCHITECTURE}

In order to ensure effectiveness of the information embedded in the compressed domain features, encoder needs to consider accuracy of compressed domain features in representing video content. The proposed encoder achieves that in two steps, as illustrated in Fig. 1. First, frame content features are extracted for each frame, in contrast to conventional block level encoder processing. Next, these content features are used as the reference for content feature accuracy within encoder parameter selection.

\section{A. Learn Object and Camera motion-models}

In [9], we used an estimate of pixel trajectories across the sequence of images, i.e. optical flow, as an estimate of motion. However, optical flow algorithms typically use an under determinant system, solved over an integration window [10], assuming locally uniform motion. This leads to blurred motion boundaries in the presence of multiple moving objects or moving camera. Motion vector refinement methods, for example the method proposed by Sung-Hee et al. in [11], typically alter MVs estimated by block matching with the objective of reducing distortion in the reconstructed image. Distortion driven methods overlook actual motion in partly moving blocks and low textured regions. Therefore, these methods are not suitable for our objective.

In order to account for multiple motions, we model frame motion as a set of moving objects, with camera motion taken as a single object with motion. Motion of each object is modelled using two dimensional six-parameter affine motion model given in (1).

$$
\left[\begin{array}{l}
V_{x} \\
V_{y}
\end{array}\right]=\left[\begin{array}{l}
a_{1}+a_{2}\left(x-x_{0}\right)+a_{3}\left(y-y_{0}\right) \\
a_{4}+a_{5}\left(x-x_{0}\right)+a_{6}\left(y-y_{0}\right)
\end{array}\right]
$$

where $\left(x_{0}, y_{0}\right)$ is the reference point for the region under consideration. The model parameters account for rotational and zooming effects as well as the translational movements. Each object model consists of motion model parameters to describe its motion and an object mask. Object models are learnt for each frame before the encoder parameter selection in the reference information pre-processing module as illustrated in Fig. 1. The algorithm consists of three main stages as illustrated in Fig. 2.

\section{Stage 1: Initialization}

For each pair of frames $F^{t+1}, F^{t}$, optical flow from frame $F^{t+1}$ to $F^{t}$, is calculated as the initial estimation of frame activity. Colour-based spatial segmentation is used to obtain an initial estimation of object boundaries in $F^{t+1}$. We denote this initial object map by $S_{0}^{t+1}=\left\{R_{0}^{t+1}, R_{1}^{t+1} \ldots R_{k}^{t+1} \ldots R_{K}^{t+1}\right\}$, where $R_{k}^{t+1} \in F^{t+1}$ is the $k^{t h}$ region. In the practical implementation, LK optical flow algorithm [12] was used to estimate motion and two-pass connected component analysis (CCA) was used for colour segmentation. 


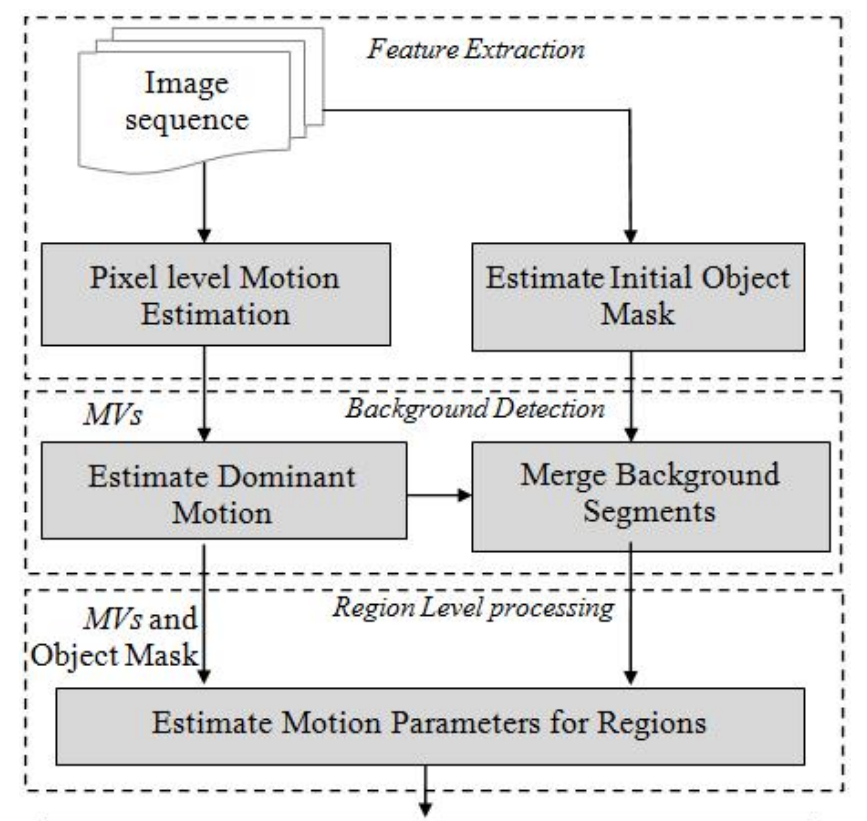

Object Map \& Expected Object Motion

To the Video Encoder

Fig. 2. Overview of Feature Extraction Process

Stage 2: Learn background and object motion models

Background region, denoted $B_{0}^{t+1}$, may contain unconnected regions depending on the layout of the foreground objects across the image plane. Therefore, it is necessary to first identify all segments corresponding to background. It is assumed that the dominant motion pattern observed in the calculated optical flow field is a result of camera movement. Therefore, six parameter affine model given in (1) is first used to estimate the frame global motion, with the reference point $\left(x_{0}, y_{0}\right)$ taken as the centre of the frame. Model parameters are estimated using iterative least square approximation with rejecting motion vectors resulting in a higher residual than an estimated threshold. The residual $D$ at a given MV is calculated using the Euclidean distance between the actual and estimated vectors. Once the model parameters are stabilized, background $B_{0}^{t+1}=\cup R_{k}^{t+1}$ is formed subject to a set of rules based on a Similarity factor, $S F(k)=|D| /\left|R_{k}^{t+1}\right|$. Here, $D$ is the set of block MV residuals defined by,

$$
D=\left\{n / n \in\|D(p)\| \triangleleft D_{\vartheta} ; p \in R_{k}^{t+1}\right\}
$$

and $|D|$ is the cardinality of the set. In the practical implementation, the least count of motion estimation in pixel units was taken as the residual threshold $D_{\vartheta}$. The value of $S F(k)$ quantifies the factor of elements identified as background blocks within a given region. Background was labelled based on the following rules.

1) Any $R_{k}^{t+1} \in B_{0}^{t+1}$, if $S F(k) \triangleright \vartheta_{u}$, and the feature vector $I_{k}^{t+1}$ associated with $R_{k}^{t+1}$ is flagged as a feasible feature value for the background.

2) Else, $R_{k}^{t+1} \in B_{0}^{t+1}$, if $S F(k) \triangleright \vartheta_{l}$ AND $\operatorname{argmin}_{\forall n \in F l a g g e d\left(I_{n}^{t+1}\right)}\left\|I_{k}^{t+1}-I_{n}^{t+1}\right\| \triangleleft I_{t+1}$

First rule accounts for measurement noise in motion estimation by considering a higher threshold. Regions adjacent with foreground objects tend to have a lower $S F$ value due to erroneous motion values at the boundary. These are accommodated in the second rule by a lower threshold supported by pre-identified background features. The feature vector consists of colour features estimated at the initial colour segmentation. In the implementations $\vartheta_{u}$ and $\vartheta_{l}$ were empirically selected as 0.8 and 0.35 .

Once frame background is identified, motion parameters of each of the remaining foreground regions are estimated by least square approximation, with the centre of each region taken as reference point $\left(x_{0}, y_{0}\right)$.

\section{Stage 3: Derive block-based motion}

Once the model parameters are learnt, object labels are assigned for each block, with priority given to foreground objects. A set of MVs for each block is calculated using the affine model and the relevant model parameters. In order to avoid blurred motion boundaries, instead of averaging pixel MVs to get the block MV, median MV is selected.

\section{B. Feature Accuracy Constrained Motion Selection}

Instead of directly using the estimated motion information to encode the video, the estimated block MVs are used as an additional constrain function, along with the encoder RD optimization. The objective of a typical encoder parameter selection is to achieve the optimum trade-off in bit rate and distortion. In the reference software for H.264/AVC [13] and H.263 Lagrangian optimization is used in encoder parameter selection [14], which forms an unconstrained optimization. If $m$ denotes a candidate vector within the search region, the optimization function is given in (2).

$$
m(\lambda)=\operatorname{argmin}_{m}\left\{D_{S A D}(m)+\lambda R_{M}(m)\right\}
$$

In ( 2) the number of bits to encode $m$ is given by $R_{M}(m)$ and $D_{S A D}(m)$ denote the estimated distortion that would result from using $m$ to displace the coding block. $\lambda$ is the Lagrange parameter. We extend the rate-constrained optimization given in (2) by introducing the objective function $\rho(m) . \rho(m)$ is modelled as the likelihood function of the candidate motion vectors, $m$, given by the Euclidean distance with the corresponding reference MV estimated in Section III $A$. Then the conventional optimization extends to,

$$
m(\lambda)=\operatorname{argmin}_{m}\left\{D_{S A D}(m)+\lambda R_{M}\right\} \text { given } \rho(m) \leq \delta
$$

where the affordable accuracy error is given by $\delta$, which effectively limit the search range to a region centred at the actual MV with distance $\delta$. Results are presented for $\delta$ in 0.5 1.5 pixel unit range. 


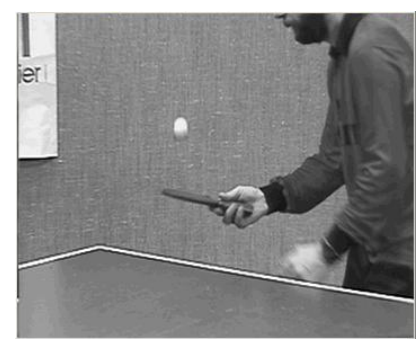

(a)

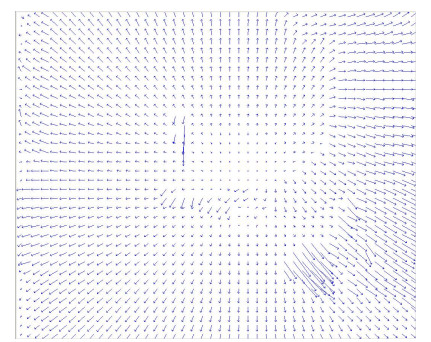

(b)

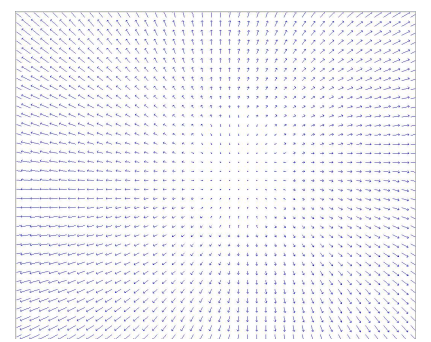

$(c)$

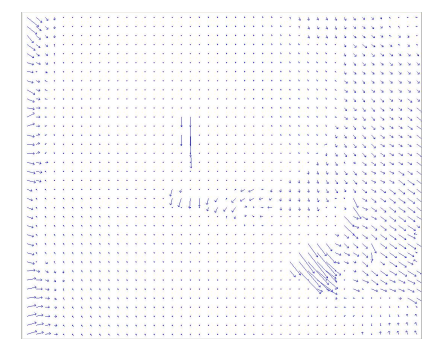

$(d)$

Fig. 3. Object detection in the presence of camera motion frame \#42 from 'Table tennis' sequence using motion vectors output of proposed method (a)Original Frame (b) Motion vector field (c) Estimated camera motion (d) Residual (object) motion

\section{EXPERIMENTAL RESULTS}

The proposed architecture considers accuracy of motion vectors in content description along with RD performance in the motion estimation process. We validate the system based on three criteria in comparison with the sate-of-theart video encoder: Performance of a vision application that uses compressed domain motion information, Complexity of MV selection process and RD performance. Furthermore, we compare the performance of motion estimated using motion vector refinement method outlined in [11] in the application scenario, with that of the proposed encoder.

Simulations were carried out using standard ITU-T standard test sequences with a range of object and camera motion properties, consisting of camera pan, camera zoom and steady camera as outlined in Table I. Sequences were in CIF (352 x 288) resolution, 4:2:0 sub-sampling format. The proposed framework was compared against the state-of-theart H.264/AVC [15] codec using JVT reference software, JM version 15.1 [13]. All sequences were encoded with an initial I-frame and a sequence of P-frames selected as the GOP structure. Frame referencing was limited to one precoded frame. Since we do not address the encoder parameter selection for block mode decision in the scope of this work, all sequences were encoded using fixed $8 \times 8$ block partition.

TABLE I

Properties of Test SEQuences

\begin{tabular}{|c|c|c|}
\hline Test Sequence & Object Motion & Camera Motion \\
\hline Soccer & High & steady camera / pan \\
Coastguard & Low & camera pan \\
Table tennis & Medium-High & zoom out \\
\hline
\end{tabular}

\section{A. Application Scenario: Background Detection and Object Segmentation}

Frame background detection, using only compressed domain MVs as cues to identify dominant motion, was considered as the first application scenario. Background detection in the presence of camera motion using MVs selected by the proposed encoder at QP 30 and $\delta=1$ for frame \#42 in 'Table Tennis' sequence is outlined in Fig 3. It can be observed that the resultant motion field is spatially consistent in the local neighbourhood. Outlier MVs at the frame edges are a result of new object appearances caused by camera zoom out. In order to analyse performance quantitatively, individual blocks classified as background were compared with manually marked ground truth. We define the percentage error in background classification as,

$$
\text { Error } \%=\frac{F P+F N}{\text { Total \# of Blocks }} \times 100
$$

where, FP and FN denote false positive and false negative detections compared to ground truth. Fig. 4 and Fig. 5 illustrate the accuracy of background detection, for 'Soccer' and 'Table tennis' sequences for $\delta$ varying from $0.0-1.5$ pixels.



Fig. 4. Avg. percentage error in background classification for 'Soccer' sequence

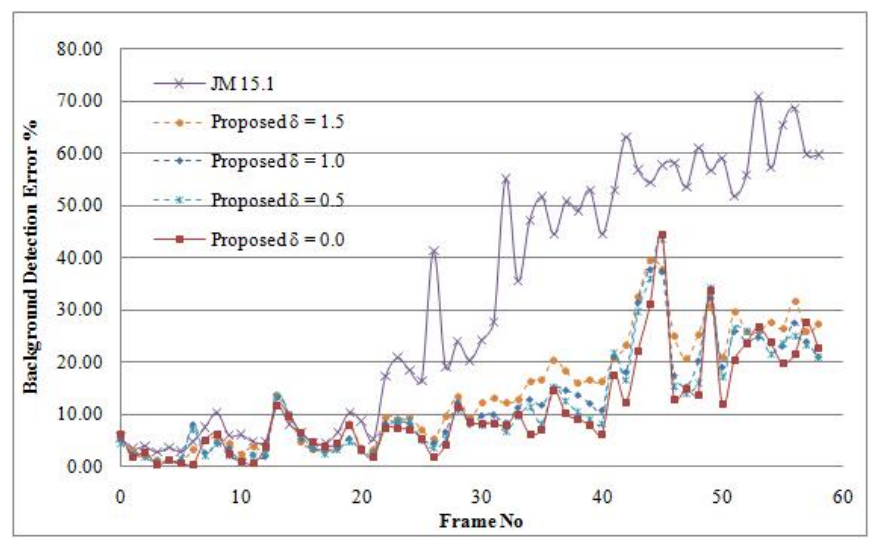

Fig. 5. Avg. percentage error in background classification for 'Table tennis' sequence 


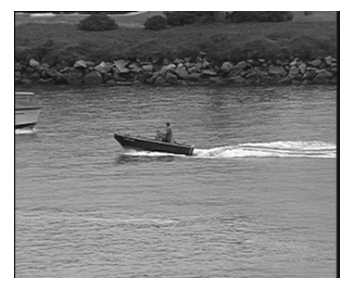

(a)

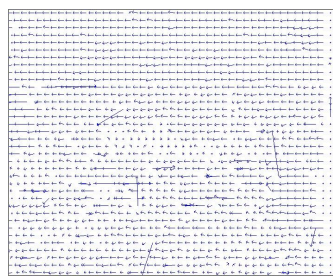

(b)



$(c)$

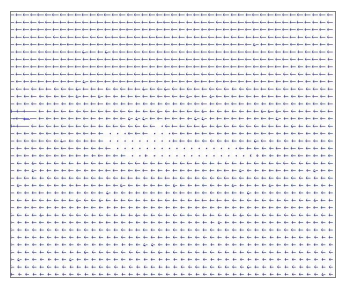

$(d)$

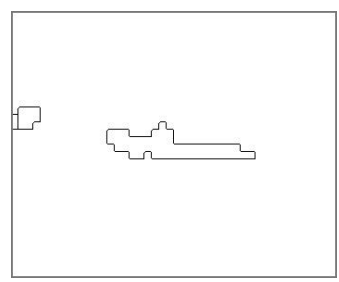

$(e)$

Fig. 6. Comparison of MVs and identified clusters for a frame in 'Coastguard' sequence (a)Original frame (b) - (c) JM reference [13] (d) - (e) proposed encoder
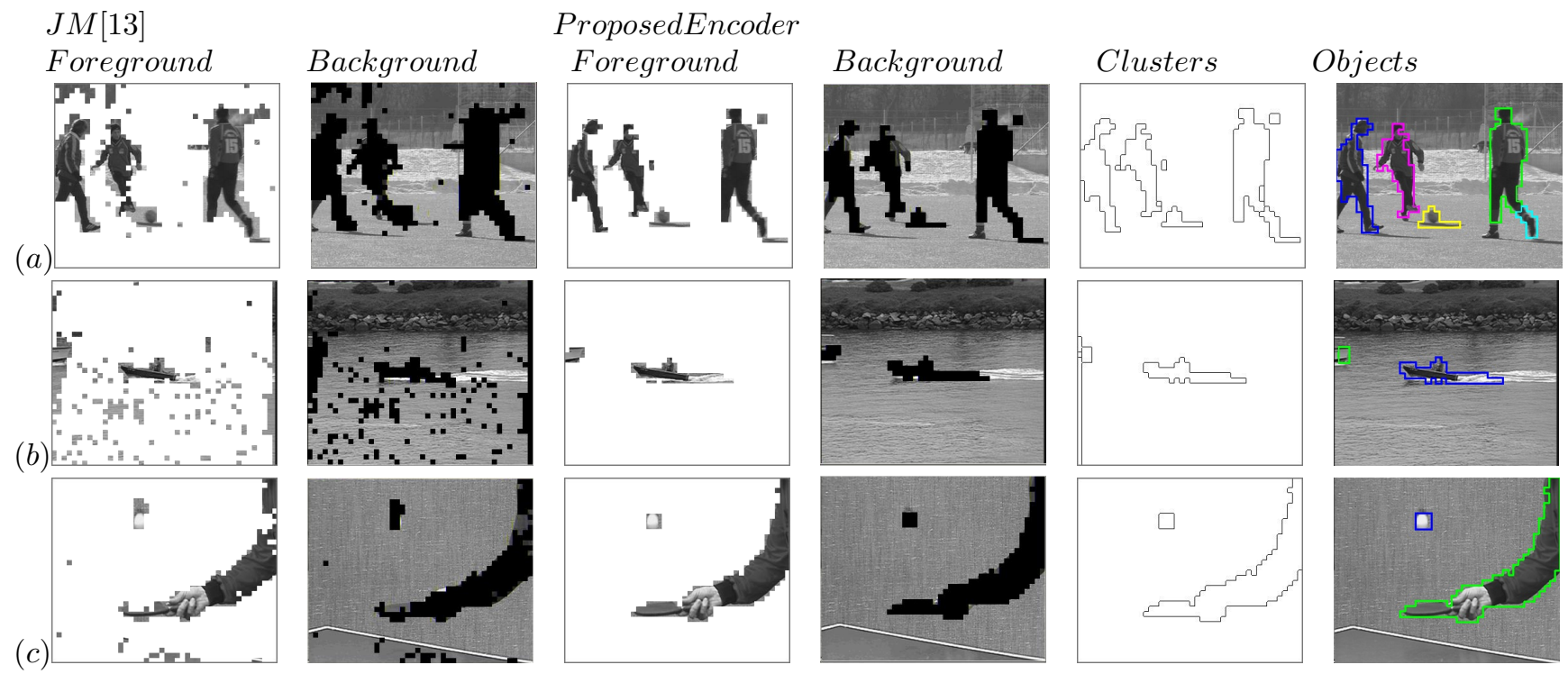

Fig. 7. Comparison of Background detection using MVs selected by JM and the proposed encoder and object segmentation using proposed encoder output for (a) 'Soccer', (b) 'Coastguard' and (b) 'table tennis' sequences

TABLE II

AVERAGE PERCENTAGE ERROR IN BACKGROUND CLASSIFICATION

\begin{tabular}{|c|c|c|c|c|}
\hline \multicolumn{2}{|l|}{ Method } & Soccer & TT & CoastGuard \\
\hline \multicolumn{2}{|c|}{ Filtered SAD [11] } & 23.73 & 45.36 & 17.78 \\
\hline \multicolumn{2}{|c|}{ JM reference [13] } & 10.43 & 32.06 & 13.45 \\
\hline \multirow{4}{*}{ Proposed Encoder } & $\delta=1.5$ & 5.52 & 14.40 & 8.10 \\
\hline & $\delta=1.0$ & 3.68 & 12.49 & 4.76 \\
\hline & $\delta=0.5$ & 3.45 & 11.85 & 4.59 \\
\hline & $\delta=0.0$ & 3.38 & 10.97 & 5.34 \\
\hline
\end{tabular}

In Fig. 4 and Fig. 5 the proposed encoder output with $\delta$ 0.51.0 demonstrate acceptable accuracy compared with $\delta=0.0$. Subjective results of background detection is given in Fig. 7. Table II outlines the average detection error in comparison with JM reference and block matched MV refinement. Relatively high error percentages in both reference methods suggest that, while these techniques achieve optimum image quality, those methods are not suitable for our purpose.

Foreground classified MVs were then used to segment moving objects using a simple connected component analysis algorithm. Fig. 6 compares the performance when MV estimated using the conventional encoder and proposed encoder are used in object detection for a frame in 'Coastguard' sequence. Fig. 7 illustrates the initial clusters and final detected objects using the MVs selected by the proposed system. Different objects are marked by colour boundaries. Clustering using MVs estimated based on object motion, is relatively accurate in all sequences.

\section{B. Complexity Analysis}

The encoder pre-processing consists of three main computations; Optical flow estimation, segmentation and least square estimation for model parameter learning. Segmentation uses connected-component analysis (CCA) assuming 8connectivity and cluster analysis, where the dominant component is the 4 comparisons made for each element. The resultant complexity is of $O(p)$, where $p$ is the number of segmented elements. The least square estimation handles $2 \times 2$ matrices. Therefore, it's complexity is of $O\left(n^{2} \times p^{2}\right)$, where $n$ is the number of warp elements which is 2 for translational motion, and $\dot{p}$ is the number of motion vectors. Practically $p$ was selected as a factor of $p$ since it is not necessary to calculate motion for each pixel as only block motion will be used. Complexity of pyramidal optical flow estimation for a single element is of $O\left(n^{2} \times L \times N^{2}\right)$, where $L$ is the number of pyramidal levels used. The dominant factor is $N$, the width of the integration window. In the implementation 3 levels were used and $\mathrm{N}$ is equal to 6 , which yields effective search range of 42 given by [12]. Therefore, overall complexity of optical flow estimation is of $O\left(\dot{p} \times n^{2} \times L \times N^{2}\right)$, which is the most 
dominant component in the pre-processing where $N^{2}$ is the most significant element. This is comparable with the typical encoder motion estimation complexity of $O\left(\check{p} \times n^{2} \times N^{2}\right)$ resulting from SAD calculations as Search range, since $N^{\prime}>>$ $N$ because of the pyramidal implementation of optical flow algorithm. Default search range, $N$ is 32 . Since the search range was restricted using $\delta$ (in the range of $0.5-1.5$ ) the computational overhead within the encoder in the proposed framework were kept is minimal.

\section{Rate Distortion Performance}

Since the proposed motion estimation process might select MVs that are not optimal for compression, RD performance can degrade compared with that of JM reference encoder. This is significant at $\delta=0.0$. However, Fig. 8 illustrates that the RD cost incurred for the 'Soccer' sequence encoded are acceptable at search ranges $(\delta) 0.5-1.0$ pixels. The RD cost can be weighed against the reduced processing required for video analysis. Other sequences also demonstrate similar behaviour.

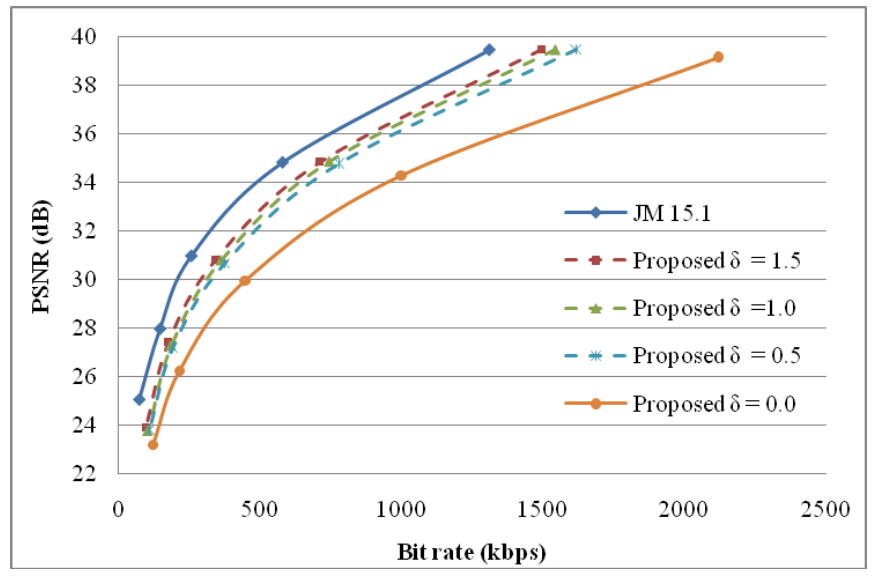

Fig. 8. Rate Distortion performance comparison for 'Soccer' sequence

\section{REAL-WORLD APPLICATIONS}

The proposed framework is particularly suitable for time critical video processing scenarios; processing in low power end-user devices. For example, in an automated multiplecamera surveillance network, reliable compressed domain features could simplify analysis at the control unit, where a large number of images are handled in real-time. Since a given camera has to parallel-process only a few frames at a time, complexity overheads at the encoder would not be significant. Similarly, in low power end-user devices such as mobile phones, one-time content driven encoding at capturing can accelerate multiple applications and processing requirements. Other applications include search, retrieval and copy detection functions in video database management, broadcast video analysis, and event detection in sports video sequences.

\section{CONCLUSIONS}

In order to optimize the performance of receiver-end video processing in a resource-limited scenario, this paper proposes a novel encoder architecture that addresses requirements of video processing applications at the encoder. An object-based motion estimation mechanism using local correlation of motion and object boundaries is proposed to enhance the quality of compressed domain motion information, with respect to its effectiveness in motion-based vision applications. Resultant motion vectors are then used to steer the encoder optimization towards selecting MVs that model actual object motion. Since this does not alter coded data structure, the decoder remains unchanged, while any application using the coded video can benefit, from this architecture. In a object detection and segmentation application scenario it is demonstrated that the resulting motion vectors can be directly used to extract object information, unlike conventionally estimated MVs based on compression efficiency.

In order to provide complete flexibility to operate the encoder at required rate, distortion as well as feature accuracy levels, the constrained optimization proposed in this work need to be extended to an unconstrained objective function. This will be addressed in the future.

\section{REFERENCES}

[1] Li H. and Ngan K. N., "Automatic video segmentation and tracking for content-based applications," Communications Magazine, IEEE, vol. 45 , no. 1 , pp. $27-33,2007$.

[2] Jin S. H. and Ro Y. M., "Video event filtering in consumer domain," Broadcasting, IEEE Transactions on, vol. 53, no. 4, pp. 755 -762, 2007.

[3] R.V. Babu, K.R. Ramakrishnan, and S.H. Srinivasan, "Video object segmentation: a compressed domain approach," Circuits and Sys. for Video Tech., IEEE Transactions on, vol. 14, no. 4, pp. 462 - 474, 2004.

[4] V. Mezaris, I. Kompatsiaris, N.V. Boulgouris, and M.G. Strintzis, "Realtime compressed-domain spatiotemporal segmentation and ontologies for video indexing and retrieval," Circuits and Sys.for Video Tech., IEEE Transactions on, vol. 14, no. 5, pp. 606 - 621, 2004.

[5] Porikli F., Bashir F., and Huifang Sun, "Compressed domain video object segmentation," Circuits and Sys. for Video Tech., IEEE Transactions on, vol. 20 , no. 1 , pp. $2-14,2010$.

[6] S. De Bruyne, W. De Neve, K. De Wolf, D. De Schrijver, P. Verhoeve, and R. V. de Walle, "Temporal video segmentation on h.264/avc compressed bit streams," The Int. Multimedia Modelling Conference, 2007.

[7] S.K. Kapotas and A.N. Skodras, "A new data hiding scheme for scene change detection in h.264 encoded video sequences," in Multimedia and Expo, 2008 IEEE International Conference on, 2008, pp. 277 -280.

[8] C. Kas and H. Nicolas, "Joint global motion estimation and coding for scalable h.264/svc high-definition video streams," in Content-Based Multimedia Indexing, International Workshop on, 2008, pp. 433 -438.

[9] R.M.T.P. Rajakaruna, W.A.C. Fernando, and J. Calic, "Facilitating motion-based vision applications by combined video analysis and coding," in Acoustics Speech and Signal Processing (ICASSP), 2010 IEEE International Conference on, 2010, pp. $1102-1105$.

[10] B.D. Lucas and T. Kanade, "An iterative image registration technique with an application to stereo vision," Proceedings of Imaging understanding workshop, pp. 121-130, 1981.

[11] Sung-Hee Lee, Ohjae Kwon, and Rae-Hong Park, "Motion vector correction based on the pattern-like image analysis," Consumer Electronics, IEEE Transactions on, vol. 49, no. 3, pp. 479 - 484, 2003.

[12] J. Y. Bouguet, "Pyramidal implementation of the lucas kanade feature tracker," Intel Corporation, Microprocessor Research Labs, 2000.

[13] G. Sullivan, T. Wiegand, and K. Lim, "Joint model reference encoding methods and decoding concealment methods," Document JVT-IO49, San Diego, 2002.

[14] T. Wiegand, H. Schwarz, A. Joch, F. Kossentini, and G.J. Sullivan, "Rate-constrained coder control and comparison of video coding standards," Circuits and Systems for Video Technology, IEEE Transactions on, vol. 13, no. 7, pp. 688 - 703, 2003.

[15] D. Marpe, T. Wiegand, and G.J. Sullivan, "The h.264/mpeg4 advanced video coding standard and its applications," Communications Magazine, IEEE, vol. 44, no. 8, pp. $134-143,2006$. 